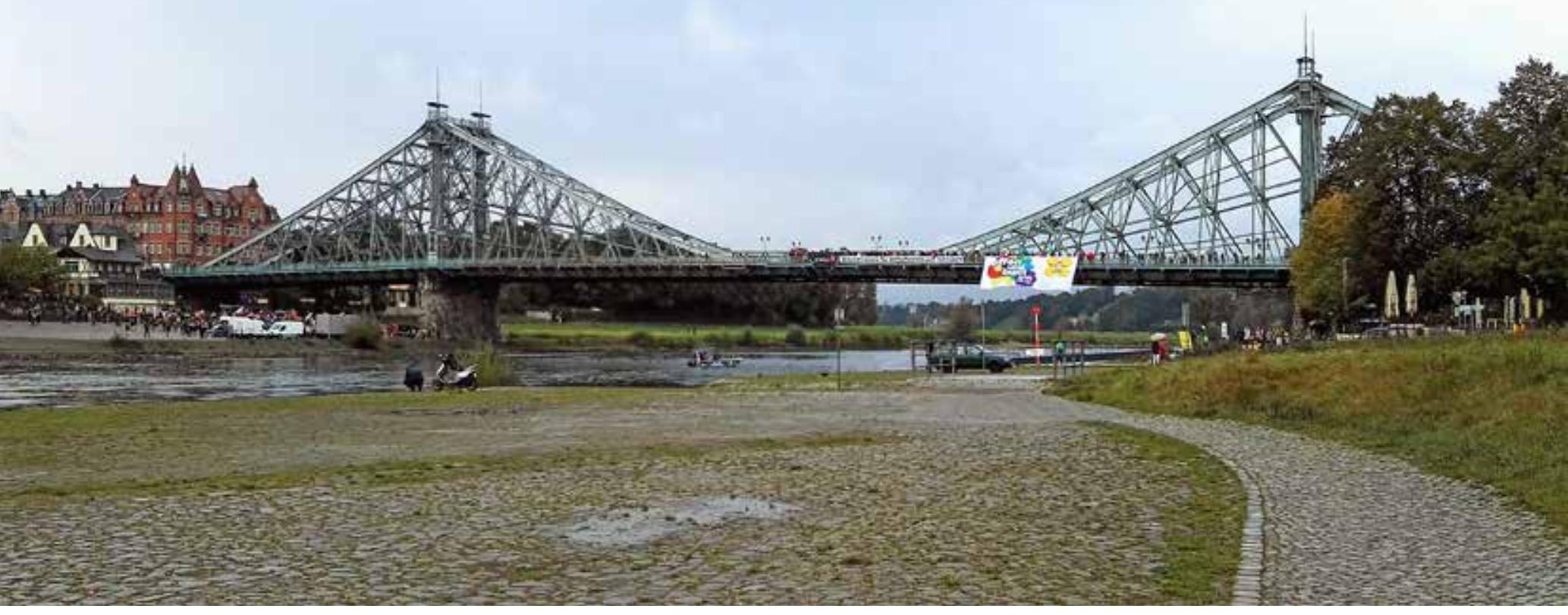

\title{
Gegen-Demokratie auf Sächsisch
}

\author{
André Brodocz, Stefanie Hammer
}

Sachsens Bürgerinnen und Bürger: streitbar, normabweichend, misstrauisch

Sachsen ist ein gefährlicher Ort. Zumindest 2015 war bundesweit in Sachsen die Gefahr am höchsten, von einem Blitz getroffen zu werden. Aber nicht nur Blitze machen Sachsen gefährlich. Auch Sachsens Bürgerinnen und Bürger weichen häufiger von Normen $a b$ als in den meisten anderen Bundesländern. Abgesehen von den Stadtstaaten Berlin, Hamburg, Bremen und dem Bundesland mit den meisten Einwohnern, Nordrhein-Westfalen, hatte Sachsen im selben Jahr die meisten Ladendiebstähle je Einwohner. Bezieht man alle Straftaten mit ein, gesellt sich zwar noch Sachsen-Anhalt in diese unrühmliche Spitzengruppe dazu, doch lässt Sachsen auch in dieser Statistik noch zehn andere Bundesländer hinter sich. ${ }^{1}$

Normen werden aber nicht nur verletzt, um sich persönlich zu bereichern. Insbesondere unter Rechts- und Linksextremen ist Gewalt ein Mittel, um den eigenen politischen Willen durchzusetzen oder zumindest öffent- lichkeitswirksam Ausdruck zu geben. Auch in dieser Hinsicht sind Sachsens Bürgerinnen und Bürger aktiver als in den meisten anderen Bundesländern. Allein das bevölkerungsreichste Bundesland Nordrhein-Westfalen hat 2015 mehr rechts- oder linksextreme Gewalttaten verzeichnet als Sachsen. In keinem anderen Bundesland gab es zudem 2015 mehr gewalttätige Übergriffe gegen Flüchtlinge und Flüchtlingsunterkünfte als in Sachsen. Widerspruch bekommt in Sachsen aber auch schon der Nachbar schnell zu hören. Im Jahr 2014 hatten knapp 40 Prozent der sächsischen Bürgerinnen und Bürger einen Streit mit ihren Nachbarn - nur in Hamburg und Baden-Württemberg wurde seinerzeit mehr gestritten. ${ }^{2}$

Sachsens Bürgerinnen und Bürger machen mit ihren Mitbürgern also häufiger die Erfahrung, dass sie von Normen abweichen und durchaus auch Streit suchen. Man könnte den Eindruck bekommen, dass sie vielleicht deshalb auch ihren politischen Repräsentanten immer weniger Vertrauen entgegenbringen. Innerhalb von 20 Jahren ist etwa die Wahlbeteiligung der Sachsen bei den Wahlen zum
Gruppierungen der Gegen-Demokratie: PEGIDA-Demonstration am Blauen Wunder und Gegendemonstration auf der Brücke am Tag der Deutschen Einheit, 3. Oktober 2016

๑ Wikimedia (Z Thomas)

1 Vgl. zur Anzahl der Blitze in Deutschland nach Bundesländern im Jahr 2015 (Blitzdichte pro $\mathrm{km}^{2}$ ), Erhebung durch Siemens, Erhebungszeitraum 2015, Veröffentlichung durch siemens.com, Juli 2016; zur Häufigkeit von Ladendiebstahl (Fälle pro 100.000 Einwohner) in Deutschland nach Bundesländern im Jahr 2015, Erhebung durch Bundeskriminalamt, Erhebungszeitraum 2015, Veröffentlichung durch Bundeskriminalamt, September 2016 (Polizeiliche Kriminalstatistik, Jahrbuch 2015, Seite 242); zur Häufigkeitszahl von Straftaten (Straftaten pro 100.000 Einwohner) nach Bundesländern von 2010 bis 2015, Erhebung durch Bundeskriminalamt/ Statistisches Bundesamt; Er- 
hebungszeitraum 2010 bis 2015, Veröffentlichung durch Bundeskriminalamt, Mai 2016 (Polizeiliche Kriminalstatistik 2015, Kurzbericht, Seite 22).

2 Vgl. zur Anzahl der politisch motivierten Gewalttaten mit rechts- bzw. linksextremistischem Hintergrund in Deutschland im Jahr 2015 nach Bundesländern, Erhebung durch Bundeskriminalamt; Erhebungszeitraum 2015, Veröffentlichung durch Bundesministerium des Inneren Juni 2016 (Verfassungsschutzbericht 2015, Seite 30, 35); zur Anzahl der Übergriffe gegen Flüchtlinge und Flüchtlingsunterkünfte in Deutschland nach Bundesländern im Jahr 2015 (Stand: bis 3. September 2015), Erhebung durch Amadeu-Antonio-Stiftung, Veröffentlichung im Handelsblatt, September 2015 (Handelsblatt, Nr. 182, 22. September 2015, S. 13); zum Ranking der Bundesländer in Deutschland nach Nachbarschaftsstreit-Quote im Jahr 2014, Erhebung durch Gesellschaft für Konsumforschung, Veröffentlichung durch Focus.de, August 2014.

3 Vgl. zur Wahlbeteiligung bei den Europawahlen in Deutschland von 1979 bis 2014 nach Bundesländern, Erhebung durch Bundeswahlleiter. Erhebungszeitraum 1979 bis 2014, Veröffentlichung durch Bundeswahlleiter; Mai 2014 (Endgültige Wahlbeteiligung und Verteilung der gültigen Stimmen nach Ländern, S. 1-2); zur Wahlbeteiligung bei den Landtagswahlen in Sachsen von 1990 bis 2014 , Erhebung durch Landeswahlleiter/in (Sachsen), Veröffentlichung durch wahlrecht. de; August 2014; zur Veränderung der Anzahl der Mitglieder der Parteien in Deutschland nach Bundesländern im Jahr 2015 gegenüber dem Jahr 1990, Erhebung durch Prof. Dr. Oskar Niedermayer (FU Berlin, Otto-Stammer-Zentrum), Veröffentlichung durch FU Berlin, Juli 2016 (Parteimitglieder in Deutschland: Version 2016, S- 15); zu Meinungen zur AfD in Sachsen, Erhebung durch Infratest dimap, Veröffentlichung durch ARD/Infratest dimap, August 2014 (LänderTREND Sachsen, S. 6).
Europäischen Parlament und zum sächsischen Landtag jeweils von über 70 Prozent auf weniger als 50 Prozent gefallen. Insbesondere bei den Landtagswahlen war die Wahlbeteiligung in den letzten Jahren allein in Brandenburg noch niedriger als in Sachsen. Dass Sachsens Bürger rasant das Vertrauen in politische Repräsentanten verloren haben, spiegelt sich darin, wie wenig Sachsen in Parteien politisch organisiert sind. Von 1990 bis 2015 verlor etwa die sächsische SPD 22,8 Prozent ihrer Mitglieder. Noch stärker sind die Verluste bei den Parteien, die auf Organisationen während der DDR bauen konnten: 64,8 Prozent bei der CDU, 87,9 Prozent bei der Linken und 82,3 Prozent bei der FDP. Misstrauen gegenüber den anderen Parteien ist es dann auch, warum 2014 ungefähr ein Drittel aller wahlberechtigten Sachsen in der AfD eine wichtige Partei sahen. Dies betrifft vor allem ihr Misstrauen in die etablierten Parteien, nicht alle Wähler zu repräsentieren, nicht alle Themen anzusprechen und nicht offen auszusprechen, was die Mehrheit denkt. ${ }^{3}$

Dieses Misstrauen lässt sich kaum mit sozialstrukturellen Merkmalen erklären, die in der sächsischen Bürgerschaft wesentlich stärker als anderswo in Deutschland ausgeprägt sind. So war etwa 2016 der Anteil an Langzeitarbeitslosen in Sachsen zwar etwas höher als im Bundesdurchschnitt, doch war deren Anteil in Bremen, Brandenburg, Nordrhein-Westfalen und Sachsen-Anhalt noch höher. Zusammen mit Sachsen-Anhalt finden wir hingegen in Sachsen mit ca. 25 Prozent den höchsten Anteil an Mitbürgern, die 65 Jahre oder älter sind, was sicherlich auf den in Sachsen und den anderen vier neuen Bundesländern geringen Anteil von ca. 4 Prozent an ausländischen Mitbürgern zurückzuführen ist. Der hohe Anteil an älteren Bürgern und Bürgerinnen scheint allerdings weniger die Lust am Streit, das normabweichende Verhalten oder das Misstrauen zu erklären als vielmehr den TV- und YouTubeKonsum: So wurde 2014 in Sachsen mehr ferngesehen als sonst in Deutschland, während wir dort bundesweit die wenigsten YouTube Nutzer finden. ${ }^{4}$

Das Misstrauen unter den Bürgerinnen und Bürgern gegenüber den politischen Repräsentanten und Institutionen lässt sich kaum darauf zurückführen, dass die Sachsen in sozialstruktureller Hinsicht anders sind als der Rest der Bundesrepublik. In Sachsen, so unsere These, beobachten wir vielmehr nur eine regionale Variante einer globalen Tendenz zur Dezentrierung demokratischer Le- gitimität, in der sich Praktiken und Institutionen der Gegen-Demokratie mehr Raum und Sichtbarkeit verschaffen.

\section{Vertrauen und Misstrauen in der De-} mokratie

Der französische Historiker und politische Theoretiker Pierre Rosanvallon hat in verschiedenen Publikationen der letzten Jahre darauf hingewiesen, dass von der wachsenden Kritik an den Parteien in den liberalen Demokratien des Westens und dem Verlust an Vertrauen in deren politische Klasse nicht vorschnell auf eine Krise der repräsentativen Demokratie geschlossen werden kann. ${ }^{5}$ Dass vor allem in der Öffentlichkeit das Misstrauen in die politischen Akteure und Institutionen als Ausdruck einer Krise debattiert wird, zeugt vielmehr von einer einseitigen Betrachtung dessen, worauf sich die Legitimität unserer Demokratien gründet. In dieser Betrachtungsweise ist die Legitimität demokratischer Institutionen exklusiv mit dem Vertrauen verbunden, dass die Bürgerinnen und Bürger ihnen schenken. Hergestellt wird diese Verbindung vor allem durch Wahlen, in denen die Staatsgewalt unmittelbar vom Volk ausgeht, indem die Bürgerinnen und Bürger diejenigen in politische Ämter einsetzen, denen sie vertrauen.

Dass der Verlust an Vertrauen in die Inhaber politischer Ämter Hand in Hand mit dem Verlust an Legitimität in die Krise führt, erscheint aber nur plausibel, wenn man allein diese Seite der Demokratie betrachtet. Demokratien beruhen aber nicht nur auf Vertrauen, sondern immer auch auf Misstrauen. Deshalb können sie ihre Legitimität auch daraus gewinnen, wie sie Legitimität mit Misstrauen verbinden. Demokratien beruhen genau genommen also auf einer Dualität von Vertrauen und Misstrauen. Diese Dualität durchzieht nicht nur die politischen Institutionen und die Art des Regierens, sondern auch das politische Engagement. Wahlen stellen die Verbindung zwischen Legitimität und Vertrauen darum auch in allen drei Hinsichten her. Mit dem Wählen verleihen die Bürgerinnen und Bürger direkt jenen Akteuren Legitimität, denen sie mehrheitlich das Regieren anvertrauen. Indem die Ausgewählten so durch Wahlen in Ämter eingesetzt werden, verleiht das Wählen unmittelbar auch Institutionen wie Parlamenten oder Regierungen Legitimität, in denen diese Ämter bereit stehen. Die Wahl verschafft den in ihre Ämter Gewählten nicht nur einen Auf- 
trag zum Regieren, sondern ebenso die Legitimität so zu regieren, wie es die WählerMehrheit von ihnen erwartet.

Wahlen haben jedoch den Nachteil, dass sie die Verbindung zwischen Legitimität und Vertrauen nur für einen Moment, die Wahl, tatsächlich leisten können. Für die Zeit bis zur nächsten Wahl sind sie nicht mehr als ein Vertrauensvorschuss der Wähler. In zeitlicher Hinsicht öffnet sich hier eine Legitimationslücke. Genau hier setzen die Verbindungen zwischen Misstrauen und Legitimität ein. Während Wahlen allerdings Legitimität und Vertrauen direkt verbinden, weil sie eben unmittelbar vom ganzen Volk ausgeführt werden, sind die Verbindungen zwischen Misstrauen und Legitimität im Vergleich dazu eher indirekt und auch deshalb weniger sichtbar.

Politische Institutionen, die nicht aus Wahlen hervorgehen, können Legitimität nur gewinnen, wenn sie dennoch dazu beitragen, den Willen des Volkes durchzusetzen oder dessen Wohl zu realisieren - und zwar gerade gegen die Regierenden, sobald diese dem in sie gesetzten Vertrauen nicht gerecht werden. Aufsichts- und Regulierungsbehörden wie etwa die Zentralbanken oder Kartellämter gewinnen ihre Legitimität aus Unparteilichkeit, indem sie Distanz zu Regierenden und Regierten wahren und sich so über Einzelinteressen zum Wohle des Ganzen hinwegsetzen können. Verfassungsgerichte hingegen können Legitimität auf Reflexivität aufbauen, indem sie die jeweils regierende Mehrheit daran erinnern, nicht mit der Gesamtheit identisch zu sein, allein weil diese Gesamtheit immer auch durch andere Mehrheiten zum Ausdruck gebracht werden könnte.

Jenseits des Wahlauftrags kann das Regieren seine Legitimität im Alltag demgegenüber auf eine Legitimität durch Nähe beziehen. Dies beinhaltet, dass im Regieren ein Interesse an den besonderen Lebenslagen aller Bürgerinnen und Bürger gezeigt und so sichtbar wird, dass niemand - insbesondere sich sozial benachteiligt, ausgeschlossen oder gar geächtet fühlende Bürgerinnen und Bürger vergessen wird und so jeder angemessen berücksichtigt erscheint. Diese Legitimität eröffnet sich dadurch, dass sich die Bürgerinnen und Bürger jenseits des Wahltags immer weniger als Mehrheits-Wahlvolk verstehen, das sich mit der Wahl selbst für einen Zeitraum an einen Auftrag gebunden hat. Vielmehr verstehen sie sich danach eher als eine Art Sozial-Volk, also als ein Volk etwa, das sich in verschiedenen, aus unterschiedlichen
Gründen vernachlässigt fühlenden Minderheiten artikuliert und auch in ihren je eigenen Formen als Volk Anerkennung beansprucht. Anerkennung finden diese Minderheiten mit einer Art des Regierens, das sich auf Nähe zu ihnen beruft. Diese Legitimität durch Nähe zu diesen Minderheiten wird deshalb dadurch gewonnen, dass sich dieses Regieren gerade auch gegen das MehrheitsWahlvolk richtet.

Sich gegen die durch Wahlen eingesetzten Politiker oder gegen den durch Wahlen hervorgebrachten Willen des Mehrheit-Volkes zu stellen, verschafft somit durchaus ebenso Legitimität wie Wahlen. Dies gilt nicht nur für bestimmte politische Institutionen oder die Art des Regierens, sondern auch für das politische Engagement der Bürgerinnen und Bürger. Politische Partizipation ist deshalb mehr als Wählen - und zwar auch in der repräsentativen Demokratie. Legitime politische Partizipation wird nicht nur durch Vertrauen, sondern auch durch Misstrauen motiviert. Das Überwachen, Anprangern und Bewerten der Regierenden sowie das Blockieren und Kontrollieren ihrer Entscheidungen sind Formen politischen Handelns, die Legitimität mit Misstrauen verbinden. Sie richten sich gegen die Legitimität, die aus Wahlen hervorgeht. Ihre eigene Legitimität gewinnen sie daraus, dass sie mit diesen Handlungen das Volk den Ursprung aller Staatsgewalt - zum Ausdruck bringen. Allerdings nimmt das Volk in Abhängigkeit von den verschiedenen Handlungen auch unterschiedliche Formen an: Es stellt sich als Wächter-Volk dar, wenn es überwacht, anprangert und bewertet, als Veto-Volk, wenn es blockiert, und als RichterVolk, wenn es kontrolliert.

Politische Institutionen, Arten des Regierens und des politischen Engagement können so also auf verschiedene Formen rekurrieren, mit denen das Volk zum Ausdruck gebracht werden kann, und deshalb auch eigene Formen der Legitimität generieren, gerade weil sie sich gegen die durch Wahlen direkt legitimierten Gewalten richten. Solche Institutionen und Praktiken bezeichnet Rosanvallon deshalb als Gewalten der Gegen-Demokratie. In den liberalen Demokratien des Westens erfahren diese gegen-demokratischen Formen der Legitimität, die das Volk als Wächter-Volk, als Veto-Volk oder als Richter-Volk bemühen, wachsenden Zuspruch, während die Wahl als Legitimität spendender Mechanismus an Kraft verliert. Zwar haben Wahlen im Vergleich zu gegen-demokratischen Formen der Partizipation den Vorteil, dass sie
4 Vgl. zum Anteil der Langzeitarbeitslosen an allen $\mathrm{Ar}$ beitslosen in den Bundesländern in Deutschland (Stand September 2016), Erhebung durch Bundesagentur für Arbeit, Veröffentlichung durch Bundesagentur für Arbeit, September 2016 (Arbeitsund Ausbildungsmarkt in Deutschland, S. 63); zum Anteil der Bevölkerung ab 65 Jahren an der Gesamtbevölkerung in Deutschland nach Bundesländern im Jahr 2014, Erhebung durch Statistisches Bundesamt; Ergebnisse auf Grundlage Zensus 2011, Veröffentlichung durch Statistisches Landesamt Sachsen-Anhalt, November 2015 (statistik.sachsen-anhalt.de); Anteil der ausländischen Bevölkerung an der Gesamtbevölkerung in Deutschland nach Bundesländern im Jahr 2015, Erhebung durch Statistisches Bundesamt, Veröffentlichung durch Statistisches Landesamt Sachsen-Anhalt, September 2016 (statistik.sachsen-anhalt.de); zur durchschnittlichen täglichen Fernsehdauer in den einzelnen Bundesländern in Deutschland im Jahr 2014 (in Minuten), Erhebung durch AGF/GfK, Veröffentlichung durch media-perspektiven.de, Oktober 2015; zum Anteil der Nutzer von YouTube an Social Media-Nutzern in Deutschland nach Bundesländern im Jahr 2015, Erhebung durch Toluna, Veröffentlichung durch faktenkontor.de, Januar 2016.

5 Vgl. zum Folgenden Pierre Rosanvallon: La contre-démocratie. La politique à l'âge de la défiance, Paris 2006; ders.: La Légitimité démocratique. Impartialité, réflexivité, proximité, Paris 2008 (dt. Demokratische Legitimität. Unparteilichkeit - Reflexivität - Nähe. Hamburg 2010). 
6 Vgl. Hans Vorländer/Maik Herold/Steven Schäller: PEGIDA. Entwicklung, Zusammensetzung und Deutung einer Empörungsbewegung. Heidelberg 2016; Oliver Decker/Johannes Kiess/Elmar Brähler: Die enthemmte Mitte. Autoritäre und rechtsextreme Einstellung in Deutschland. Die Leipziger "Mitte“-Studie 2016. Gießen 2016.

7 So etwa Lars Geiges/Stine Marg/Franz Walter: PEGIDA. Die schmutzige Seite der Zivilgesellschaft? Bielefeld 2015. verschiedenen Dimensionen des politischen Engagements bündeln, indem sie gleichzeitig politischen Willen ausdrücken, die Teilhabe an der Gesetzgebung durch die Auswahl der Repräsentanten ermöglichen und durch die Abwahl einer Regierung auch in den politischen Prozess eingreifen lassen, während gegen-demokratisches politisches Engagement häufig nur eine Dimension bedient. Das Mehrheits-Wahlvolk hat allerdings auch Nachteile: anders als das Wächter-Volk kann es die Regierenden nur im Moment der Wahl überwachen; anders als das Veto-Volk braucht es gemeinsame Ziele oder Motive, um eine Regierungsmehrheit zu schmieden, was für das bloße Blockieren einer einzelnen Entscheidung nicht nötig ist; und anders als das Richter-Volk, das im Einzelfall über die politische Klasse urteilen kann, ist die Wahl zunächst nur eine Entscheidung, deren Folgen im Einzelnen noch nicht absehbar sind.

Stellt man diese Entwicklungen in Rechnung, dann erleben wir gegenwärtig eine globale Tendenz zur Dezentrierung demokratischer Legitimität, mit der Ambivalenzen und Probleme einer sich allein durch Wahlen legitimierenden demokratischen Ordnung sich möglicherweise überwinden lassen.

\section{Ambivalenzen der Gegen-Demokratie}

Die jüngere Geschichte der politischen Kultur Sachsens lässt sich vor dem Hintergrund der Dezentrierung demokratischer Legitimität als eine regionale Ausprägung dieser weltweit zu beobachtenden Tendenz betrachten. Dadurch wird auch deutlich, dass das verbreitete Misstrauen gegenüber den Institutionen der Wahl-Demokratie und der damit verbundene gegen-demokratische Aktivismus nicht zwingend als Legitimitätsverlust der demokratischen Ordnung angesehen werden müssen, sondern dazu beitragen können, der Demokratie andere Legitimitätsressourcen zu erschließen. Allerdings sind wir damit ebenso wenig Zeugen eines demokratischen Fortschritts, weil auch die gegen-demokratischen Institutionen und Praktiken selbst nicht frei von Ambivalenzen sind.

So weist auch Rosanvallon darauf hin, dass gegen-demokratisches Engagement selbst zwar nicht unpolitisch ist, aber unpolitische Auswirkungen haben kann. Zum einen sind gegen-demokratische Praktiken zuerst darauf angelegt, von einer Mehrheit geteilte Vorstellungen über die gemeinsam angestrebten Ziele in Frage zu stellen. Da gegen-demokra- tische Praktiken auf diese Weise stets nur reaktiv wirken, tragen sie selbst zur weiteren Entwicklung solch kollektiv angestrebter Projekte nichts bei und lösen diese so perspektivisch auf. Zum anderen tragen gegendemokratische Praktiken dazu bei, die Fragmentierung der Zivilgesellschaft weiter voranzutreiben. Dadurch können die direkt demokratischen Institutionen allerdings auf Dauer genau den sozialen Ort verlieren, an dem öffentlich die Probleme als gemeinsame Aufgaben identifiziert werden, um deren Lösung die Repräsentanten mit verschiedenen Programmen um die Mehrheit der Wähler konkurrieren.

Vor allem in Sachsen wurden diese Ambivalenzen in den letzten Jahren sichtbar. Die historischen Plätze der sächsischen Hauptstadt werden hier regelmäßig am ebenso historisch bedeutsamen Montag auf der einen Seite durch die „Patriotischen Europäer gegen die Islamisierung des Abendlandes“ (PEGIDA) sowie auf der anderen Seite von Vertretern der NoPegida-Bewegung besetzt. Was PEGIDA und NoPegida wöchentlich zusammenbringt, ist die öffentliche Inszenierung beider Bewegungen: das Aufsuchen eines öffentlichen Ortes, um die jeweils eigenen Forderungen, mithilfe performativer Elemente, zum Ausdruck zu bringen. Es sind jedoch die Inhalte dieser Forderungen, die die beiden Gruppen unterscheiden. So richtet sich der Protest von PEGIDA nicht allein gegen die Asylpolitik der Bundesregierung, sondern vornehmlich gegen deren Mitglieder, die als „Volksverräter“ beschimpft und „an den Galgen“ gewünscht werden, während die symbolischen Forderungen von NoPegida „Kein Mensch ist illegal“ oder „Refugees welcome“ inklusiv formuliert sind.

Die sächsischen Anhänger PEGIDAs, soviel scheint bei aller Verschlossenheit gegenüber Außenstehenden sicher, sind mehrheitlich männlich, biographisch in der mittleren Lebensphase zwischen 30 und 60 Jahre alt und gehören keiner Religionsgemeinschaft an. Ihre politischen Einstellungen zeichnen sich durch Misstrauen und Distanz gegenüber der bundesdeutschen Demokratie und deren etablierten politischen Institutionen aus. Während die Zustimmung zur „Demokratie als Idee“ in der bundesdeutschen Bevölkerung in den letzten zehn Jahren immer bei über 90 Prozent lag, liegt sie bei denjenigen, die mit den Zielen von PEGIDA vollkommen übereinstimmen, bei unter 70 Prozent. Und auch wenn schon im Verhältnis zur sächsischen und erst recht zur bundesdeutschen Gesamt- 
bevölkerung stets nur eine Minderheit an den Demonstrationen von PEGIDA teilnimmt, so nehmen sie doch wie selbstverständlich für sich in Anspruch, für das deutsche Volk zu sprechen. ${ }^{6}$

NoPegida umfasst als politische Bewegung eine Vielzahl zivilgesellschaftlicher Gruppierungen und Netzwerke, deren kleinster gemeinsamer Nenner die Gegnerschaft zu PEGIDA ist. Sie nennen sich Dresden Nazifrei, Bündnis für ein offenes Dresden und stellen dem Anspruch PEGIDAs auf Volksvertretung ihr „\#ihrseidnichtdasvolk“ entgegen. Manch einer erkennt darin schon die funktionierende, helle Seite der Zivilgesellschaft. ${ }^{7}$ Tatsächlich wissen wir aber bei NoPegida noch weniger über die Motive der einzelnen Akteure als im Falle der Pegidisten. Bei einer politischen Bewegung, die sich aus Antifa, lokalen politischen Bündnissen und regionalen Initiativen zusammensetzt, kann aber angenommen werden, dass die Einstellungen zu Staat und Demokratie hier durchaus weit auseinandergehen können.

Und so lässt sich mit Rosanvallon zunächst nur resümieren, dass wir im Falle Sachsens von (mindestens) zwei Gruppierungen einer Gegen-Demokratie sprechen können, die sich nicht nur unversöhnlich gegenüberstehen und die Fragmentierung der (sächsischen) Zivilgesellschaft am geschichtsträchtigen Montagstermin vorantreiben. Sie führen uns auch vor Augen, wie sich jenseits von Wahlen das Volk zunehmend durch verschiedene Minderheiten artikuliert und so die schleichende Erosion des Wahl-Volks als Ursprung aller Staatsgewalt betreibt. So auch am 3. Oktober 2016, als in Dresden die jährliche Einheitsfeier stattfand. Am 26. Jahrestag dieses Ereignisses wollte Sachsen, als Gastgeber, sich von seiner hellen Seite zeigen und - so das Motto der offiziellen Feierlichkeiten - „miteinander/zueinander/füreinander BRÜCKEN BAUEN“. Es sollte anders kommen: Während die politische Elite drinnen feierte, wurde draußen lautstark gestört, miteinander gegeneinander demonstriert und so der sächsischen Gegen-Demokratie als eine regionale Variation für die Ambivalenzen der laufenden globalen Dezentrierung demokratischer Legitimität weltweite Aufmerksamkeit beschert.

\section{Autoren}

Prof. Dr. André Brodocz Professur für Politische Theorie, Staatswissenschaftliche Fakultät, Universität Erfurt Postfach 900221 99105 Erfurt

Dr. Stefanie Hammer Wissenschaftliche Mitarbeiterin an der Professur für Politische Theorie, Universität Erfurt 